\title{
${ }^{\circledR}$ A New Approach for Modeling Dissipation due to Breaking in Wind Wave Spectra
}

\author{
Dorukhan ARdag AND Donald T. Resio \\ College of Computing, Engineering and Construction, University of North Florida, Jacksonville, Florida
}

(Manuscript received 15 July 2019, in final form 1 November 2019)

\begin{abstract}
A robust spectral dissipation term for wind waves has long been a goal of detailed-balance spectral modeling and is represented by many different approximations in spectral models of random wave fields. A Monte Carlo approach is employed here to create a random-phase sea surface that is used to simulate the distribution of horizontal surface velocities at the sea surface and to relate these velocities to deep-water wind wave breaking. Results are consistent with many recent studies that show a kinematic-based breaking criterion can provide a consistent depiction of the onset of wave breaking. This criterion is combined with the calculated nonlinear flux rates to estimate a transition point within a spectrum at which a spectrum changes from an $f^{-4}$ equilibrium-range form to an $f^{-5}$ region dominated by dissipation, potentially an important factor within several air-sea interaction mechanisms, turbulence at the sea surface, and remote sensing applications. It also has the potential to improve operational modeling capabilities.
\end{abstract}

\section{Introduction}

Wave breaking, represented in spectral models as $S_{\text {brk }}$, is a critical process at the ocean-atmosphere interface, enhancing gas exchanges between air and water and providing a vital mixing mechanism for momentum exchanges between the sea surface and the atmosphere (Melville 1996). In a physical sense, $S_{\text {brk }}$ is the least understood term among wave-model source terms. It is temporally and spatially sporadic and occurs on a much shorter time scale than other major source terms contributing to wave generation and decay (nonlinear resonant interactions and wind input) (Rapp and Melville 1990). Hence, despite numerous scientific investigations over the years, $S_{\text {brk }}$ has no universally accepted mathematical form (Elgar 2007), which is not surprising since the highly nonlinear character of wave breaking in random seas makes it very challenging to interpret $S_{\text {brk }}$ on a time-space scale commensurate with other source terms used in spectral wave models.

Presently, instrument-based observations of energy dissipation in wind waves must be supported by visual observations to develop quantitative concepts for source

Denotes content that is immediately available upon publication as open access.

Corresponding author: Donald T. Resio,don.resio@unf.edu terms (Banner et al. 2000; Babanin et al. 2001; Romero et al. 2012; Schwendeman et al. 2014). Even in this context, it is possible to locate breaking events in space but not straightforward to estimate the spectral distribution of associated energy losses in frequency and wave propagation angle.

Dynamic, kinematic, and geometric criteria have all been employed to characterize limits for incipient wave breaking mechanisms and the onset of breaking. However, the most commonly used criteria in spectral wave models today, wave steepness, does not have a well-established theoretical basis that appears consistent with observed behavior. Wave steepness in monochromatic, unidirectional waves of permanent form has been well studied for many years (Stokes 1880; Gerstner 1809). Theoretical bases for extending the steepness concept to modeled spectra can be found in Alves and Banner (2003) and within the context of narrow-banded wave spectra by Song and Banner (2002). Experimental evidence from simulations with a small number of frequencies with no directional spreading were used to support theoretical developments related to steepness in Banner and Peirson (2007). However, the conventional interpretation of wave spectra is that they are continuous in both frequency and direction (Hasselmann 1961; Zakharov and Filonenko 1967), which allows fourwave interactions to exist as a dominant source term, which served as the primary motivation for the need 
to develop detailed-balance (third-generation) wave models.

Numerical simulations and experiments based on discrete wave components have typically used a limited set of unidirectional, monochromatic frequencies to simulate energy densities in tests. Results from this type of study have been used to argue that wave breaking is related to wave steepness and that the spectral dissipation function in wave models should have its peak values in the vicinity of the spectral peak (Alves and Banner 2003). Observationally, a substantial portion of wave breaking is observed to be collocated with large waves (Banner et al. 2000). However, as will be shown here, this may not imply that most energy is being lost from the spectral peak region.

In typical wind seas, whitecaps are widespread and relatively frequent in time and space, which limits the applicability of methods based on small numbers of spectral components to explain wave breaking probabilities in space and time, even though such experiments are extremely useful for providing guidance regarding the physics governing the breaking mechanism itself. It should be noted that we are referring specifically to probabilities of wave breaking in locally generated seas in this paper and not arguing that nonlinear interactions and wave breaking at the spectrum peak are not important processes in narrowbanded spectra.

Attempts to utilize the concept of steepness-limited breaking in wave models have been somewhat heuristically founded on dimensional arguments which suggest that steepness in a directionally integrated spectrum in frequencies above the spectrum peak should be consistent with an $f^{-5}$ form, that is, $E(f)=\alpha g^{2} f^{-5}$, where $f$ is frequency, $g$ is gravity, and $\alpha$ is a dimensionless constant (Phillips 1958). This led the use of $f^{-5}$ bases in the development of many spectral shapes (Phillips 1958; Pierson and Moskowitz 1964; Hasselmann et al. 1973) up through the mid-1970s. The JONSWAP spectrum was the last major spectral form for wind waves in an active generation area that was based entirely on such a form. Since that time the majority of spectral shapes have been based on equilibrium ranges with characteristic $f^{-4}$ forms (Toba 1972; Donelan et al. 1985; Resio et al. 2004).

In this paper, we will present an argument that both of these ranges may exist simultaneously within a spectrum and that this would be consistent with the form of wave breaking that we develop here. Given that wave breaking is observed to occur in discrete time and space events at the sea surface, it seems unlikely that wave breaking can be well represented by a process that varies smoothly over the entire spectral domain. Instead, it seems more likely that an entire portion of the spectrum might change its average spectral form to reflect the addition of this new dissipation into the overall sourceterm balance. The question is whether this transition is relatively sharp or distributed over a broad range of frequencies. In either case, this shape transition must be located at a point where all energy fluxes into this region from wind inputs and nonlinear interactions must be balanced by dissipation.

In the context presented here, a probabilistic relationship for wave breaking exceeding a common breaking threshold distributed in space and time on the sea surface is developed. Solving for the mean occurrence rate (mean breaking frequency) yields an estimate of the expected transition region where discrete breaking events create a change in spectral shape. At lower frequencies $S_{\mathrm{ds}}$ is assumed to approach zero, unlike what is expected from parameterized representations of continuous spectral dissipation functions today, which are distributed continuously from the vicinity of the spectral peak to very high frequencies. Here, it is expected that the spectral shape in the region lower than the transition zone should follow an $f^{-4}$ equilibrium-range form, while at higher frequencies, the spectral form is expected to become dominated by only gravity and frequency in the breaking zone and is expected to follow an $f^{-5}$ form (Phillips 1958).

The new breaking source term developed here will be shown to be consistent with kinematic limits for incipient, sporadic wave breaking within a statistical framework for the occurrence of threshold exceedances. A critical assumption adopted here is that, although wave breaking is recognized to be related to wind speed, as noted by many empirical relationships proposed between wind speed and percentage white cap coverage, additional factors, such as wave age, may also influence this rate of breaking (whitecapping).

The objectives of this paper are 1) to synthesize results from several recent studies that support a clear basis for a specific kinematic breaking criterion related to the ratio of the energy propagation velocity (i.e., group velocity) to the currents generated by longer waves; 2) to develop a methodology to quantify the rate and spectral distribution of energy loss due to wave breaking; and 3) to show a consistency between the predicted loss rate and the nonlinear fluxes into the portion of the spectrum dominated by breaking. Following a brief review of dynamic and kinematic bases for wave breaking, this paper will devote one section to each of the three objectives noted above. Our discussion will examine the potential role of the breaking formulation introduced 
here to the development of a transition from an $f^{-4}$ to an $f^{-5}$ characteristic spectral form.

\section{Previous research on wave breaking}

Literature dedicated to wave breaking has extensively examined two essential questions: 1) what physical process leads to wave breaking (breaking onset in individual waves) and 2) what role does wave breaking play in the evolution of wave spectra within wave models related to the spectral dissipation source term? Finding an answer to the first question does not provide a straightforward explanation for the sporadic breaking observed in wind seas, especially considering the number of frequency and directional constituents and their complex flux interchanges in oceanic wave fields. An additional step is required for application to phaseaveraged wave modeling. In this paper, our intention is to address these research problems within a single coherent framework.

Perlin et al. (2013) provided an extensive analysis of numerous studies which tested and/or utilized the three breaking criteria for the onset of wave breaking (geometrical, kinematic, and dynamic). Some of these perspectives that are directly or indirectly related to our framework are reviewed here, especially the kinematic and dynamic criteria. The geometric criterion considers wave shape, typically in terms of wave asymmetry or some steepness limit. Stokes (1880) found this limit to be $(a k=0.4432)$. Laboratory studies (Rapp and Melville 1990; Tulin and Landrini 2001) showed breaking could occur at low steepness values $a k$ in the range of 0.15-0.2. Toffoli et al. (2010) combined deep water observations and laboratory measurements to show that waves could exist past this steepness limit even though they are in the breaking phase.

The kinematic criterion relates horizontal velocities at the water surface (typically occurring at or near the crest of a large wave) to the group or phase velocity of a wave. This framework limits the particle velocity at the tip of a wave crest to some fraction of the wave propagation speed. When a time-space varying current interacts with a wave, the wavelengths are stretched (diverge) as the velocity of the current increases and compressed when the current velocity decelerates. Longuet-Higgins (1988) named this the "concertina" effect. If the scale of time variation were comparable or less than the wave period of the propagating waves, we expect that this phenomenon would interact with individual waves at the scale of the phase velocity; but if it occurs on a scale equal to or greater than the wave period, this convergence should be better related to the energy propagation speed (i.e., group velocity). If the wave energy is compressed more than some threshold value, we expect the convergence to produce an instability at the surface (wave breaking).

As mentioned in Perlin et al. (2013) and several recent works including thermal image velocimetry (TIV) measurements (Saket et al. 2017); numerical simulations (Irisov and Voronovich 2011; Derakhti and Kirby 2016; Barthelemy et al. 2018); and buoy measurements (Waseda et al. 2014) which are treated in more detail subsequently in this section, the kinematic breaking limit defines a breaking criterion $U / C$ around $0.8-1$. In this work, $U$ is the horizontal surface velocity and $C$ is taken as group velocity.

The dynamic breaking criterion, described above, uses the equations of motion to locate the zones of convergence on a wave field which lead to wave breaking (Longuet-Higgins 1988; Irisov and Voronovich 2011). The complexity of translating this approach into Fourier space makes it difficult to implement in spectral models; however, the so-called concertina effect provides results that are consistent with this criterion for breaking (Longuet-Higgins 1988) and offers a physical interpretation of this process.

Duncan (1981), in an experimental procedure using a towed hydrofoil, investigated the cause of breaking and the total energy lost during a continuous breaking process. Using the tangential component of the weight of the breaking region, he came up with a $c^{5}$ scaling for breaking, where $c$ in this case was the wave celerity. This concept was utilized and tested in various ways (Phillips 1985; Rapp and Melville 1990; Romero et al. 2012); however, the application of this scaling from steady laboratory conditions to the sporadic wave breaking observed in wind waves is still considered open to question (Schwendeman et al. 2014). Additionally, breaking in the field observations must be measured using drifters or moored buoys, which can introduce observation bias and/or neglect of small-scale breaking (Romero et al. 2012; Schwendeman et al. 2014).

To investigate Duncan's experimental data with respect to kinematic breaking criteria, we calculated the ratio of the horizontal surface velocity to group velocity, using a simple linear approximation. The results are shown in the Fig. 1, where filled squares represent the breaking cases and unfilled squares are nonbreaking cases. In the figure, the kinematic breaking region, $U / C_{g} \sim 0.85$, is shown to fall midway between the breaking and nonbreaking cases, where $U$ is the surface horizontal orbital velocity and $C_{g}$ is the group velocity.

Using 2D potential motion dynamic models (Lagrangian and Hamiltonian approaches) Irisov and Voronovich (2011) were able to create a reasonable approximation to the incipient breaking stage in wave spectra by 


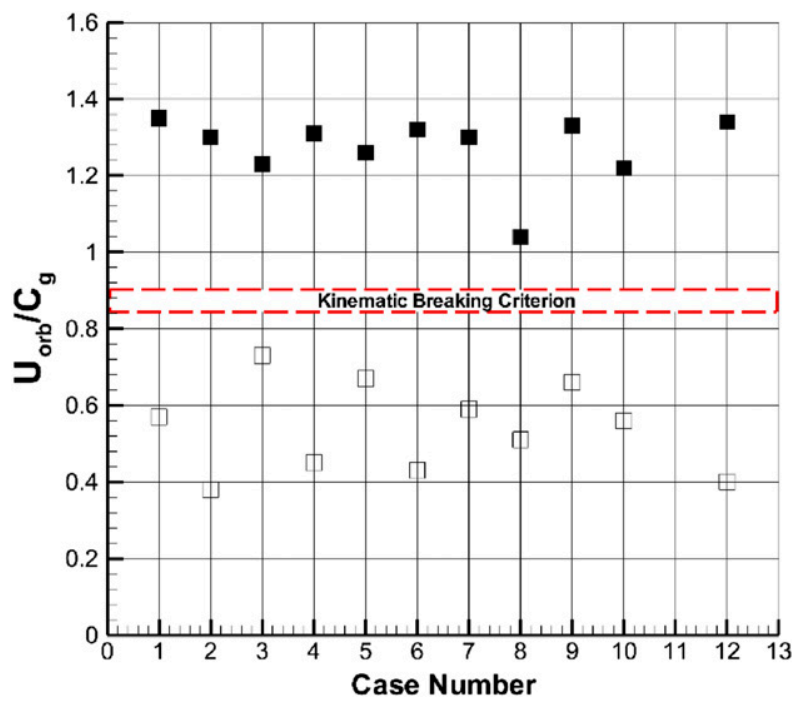

FIG. 1. The 11 test cases from Duncan's (1981) experiment. Filled squares show the breaking events whereas the empty squares represent the nonbreaking cases. A hypothetical kinematic breaking region is marked by the red dashed line.

comparing instability points on the wave surface with maximum current convergence and surface slope locations. They noted that the phenomenon modeled was similar to the concertina effect described by LonguetHiggins (1988). The correlation between the breaker position and maximum current convergence was shown to be significant, which was combined with the RMS of the convergence rates to relate it to the breaking frequency. By looking at the horizontal velocities at the steepest crest in a similar manner with Banner and Tian (1998), they suggested $U / C_{g}$ should be approximately 0.8 . The main conclusion drawn by Irisov and Voronovich (2011) was that the dominant breaking in random wave fields occurs at intermediate frequencies rather than at the spectral peak or only at very high frequencies.

A unique study by Waseda et al. (2014) recorded characteristics of the deep ocean surface waves, including extreme waves, by deploying a moored buoy and a drifting buoy. The GPS sensors on these buoys allowed them to measure the horizontal displacement on the wave surface and estimate orbital velocities. This provided a Lagrangian framework as opposed to the more conventional Eulerian observational basis. When waves move to the peak of the group, speed of the particles at the crest accelerates, even if they are not breaking (Tulin and Landrini 2001). Looking at the $U / C_{g}$ (normalized maximum horizontal particle speed) values from their deep water observations, Waseda et al. (2014) were able to show that there is a threshold (0.85-0.9) above which most waves break (Fig. 14 in their work).
Another interesting point from their work was that their ensemble average spectrum in deep water followed an $f^{-4}$ form in the equilibrium range at frequencies lower than this threshold value. The implication here is that even during low wind and/or low steepness (low breaking) conditions nonlinear interactions can keep the spectra in this form in spite of active wave breaking at higher frequencies.

In this paper, the frequency at which the stretching and convergence due to the sum of all surface orbital velocities from 0 to $f_{b}$ equals the kinematic breaking threshold is estimated within the context of wavecurrent interactions. A linearized version of this can be written as $\left\{\left[\sum_{f=0}^{f_{b}} u_{\text {orb }}(f, \theta) \cos (\theta)\right]+c_{g}\left(f_{b}\right)\right\} / c_{g}$. Itay and Liberzon (2017) performed an experiment that investigated the kinematic breaking limit of shoaling waves in a large wave flume and found that the limit of the ratio of the Lagrangian water velocity on the crest of a wave to the propagation velocity of that wave fell in the range 0.8-1.04. Since their experiment was in very shallow water (depth divided by wavelength less than $0.5)$, the phase velocity and group velocity are essentially identical in this experiment; consequently, this experiment does not disagree with the data of Waseda et al. (2014), who used the group velocity in his spectral measurements. In a wave spectrum the more general form (including depth) shown above is consistent with the data (Duncan 1981; Banner and Tian 1998; Irisov and Voronovich 2011; Waseda et al. 2014).

By employing a 3D boundary element model, Barthelemy et al. (2018) calculated normalized energy flux ratios at the tip of unidirectional chirps, noting that on the free surface and under zero surface pressure, this flux ratio reduces to a kinematic condition. The critical breaking threshold where waves start breaking was found to be related to energy fluxes when $U / C$ exceeded a value of 0.85 in shallow water; and verified this ratio as a reasonable approximation for breaking in 2D and 3D deep and intermediate depths. Saket et al. (2017) measured the kinematic ratio of unidirectional wave groups in a wave tank using thermal image velocimetry with and without wind forcing to investigate the same criterion and came up with almost identical results. Incorporating wind effects only affected this ratio by about $2 \%$. Both studies concluded that, in contrast to the consistency of the kinematic breaking criterion, the onset of breaking could not be discriminated by the steepness criterion alone.

Derakhti et al. (2018) used large eddy simulations to simulate nonlinear wave evolution focusing on breaking onset and post breaking behavior. They simulated cases for breaking over flat bottom topography with dispersive focusing and modulational instability for $2 \mathrm{D}$ and $3 \mathrm{D}$ 
cases. They were able to verify the same outcome as Barthelemy et al. (2018), that the breaking onset could be represented by using the ratio $U / C_{g}=0.85$ ( $B$ in their notation) with the breaking region occurring at the tip of the wave.

Since the kinematic criteria appear to provide the most robust representation of wave breaking, we shall utilize this approach in our transition to a dissipation source term for wave spectra. There seems to be a disparity in the literature when it comes to the use of phase speed or the group speed as the basis for the ratio shown above, depending on the numerical experiment, laboratory or even field measurements and what wave phenomenon is being investigated. Here we choose the overall modeling framework that appears to fit a consistent interpretation from several different studies. Typically, group velocity governs energy fluxes and effects of propagation speed along the axis of propagation, while refraction effects related to speed variations along a wave crest. For example, shoaling on opposing currents increase nonlinearity leading to the oversteepening of waves, which impacts the wave surface kinematics. Similarly, long waves create zones of convergence and divergence of currents can destabilize superposed shorter waves. Therefore, in a spectral basis, group velocity, which controls energy fluxes within the spectra, is chosen here for the wave speed in our breaking criterion.

Presently, steepness limits have been a fundamental basis for the dissipation source term in most operational wave models, possibly due to the convenience of applying it to wave spectra in model applications (Komen et al. 1984; Alves and Banner 2003; Babanin et al. 2010). Alternative representations for this process include weak-in-the-mean forms (Hasselmann 1974) in which breaking is linearly related to the wavenumber spectrum, prescriptive methods that utilize a saturation limit (Phillips 1985; Alves and Banner 2003) and exceedance probability formulations (Babanin et al. 2010). Recently, short-wave modulation and related breaking has found support in recent field work and subsequent theoretical work (Young and Babanin 2006; Tsagareli et al. 2010). This concept postulates that long waves distort the short waves and force convergence on the front face of the long waves, which then increases the steepness of the shorter waves, leading to breaking (Donelan 2001). This is basically an analogous concept to that proposed here.

Phillips (1958) used dimensional arguments to derive a spectral shape that incorporated wave breaking within it. Assuming that wind inputs occur on a much longer time scale than wave breaking, he proposed a dimensionally consistent equilibrium range proportional to $\alpha g^{2} f^{-5}$ with $\alpha$ being a universal coefficient equal to 0.0074 . Through the early 1970s, the $f^{-5}$ form hypothesized by Phillips was the accepted basis for the shape of wind wave spectra (Pierson and Moskowitz 1964; Hasselmann et al. 1973); however, Toba (1973) presented strong observational evidence that the shape of the energy spectra follows an $f^{-4}$ form above the peak region. This has now been confirmed by many subsequent studies (Forristall 1981; Donelan et al. 1985; Resio et al. 2004; Romero and Melville 2010; Waseda et al.2014). In several separate observational studies (Long and Resio 2007; Romero and Melville 2010; Lenain and Melville 2017), measured wave spectra were grouped according to their wave ages, which revealed a correlation between wave age to where the transition from an $f^{-4}$ form (assumed to be controlled by nonlinear energy fluxes) to an $f^{-5}$ (assumed to be breaking dominated) region happens on a normalized frequency scale $\left(f l f_{p}\right)$. This paper investigates the location of this nondimensional transition frequency (which will be referred to as $f_{\mathrm{ti}}$ ) by looking at kinematic and dynamic criteria on a random sea surface for an accurate and stable modeling basis that captures the spectral wave evolution.

\section{A new formulation for wave breaking in a spectrum}

To quantify the role of the ratio $U_{\mathrm{orb}} / C_{g}$ in wave spectra, we considered a range of sea states. In these simulations, wave records were generated at a single point by assigning initial random phases for frequency components of spectra using $f^{-4}$ equilibrium ranges similar spectral structure in Resio et al. (2011), which has an $f^{-4}$-based peak enhancement factor and a $u^{2} c_{p}$ scaling in the equilibrium range, Simulations were conducted for a range of wind speeds and peak frequencies, linearly superposing each of the spectral components. The initial conditions used $N_{\text {frq }}=191$ equally spaced frequencies between 0.05 and $1 \mathrm{~Hz}$ with the sampling interval of the sum chosen to be $1 \mathrm{~s}$. We used a simple $\cos ^{4}$ distribution for the angular spreading in these initial spectra when investigating the effects of angular variations on breaking. This simulation was executed for a range of wave age values from young seas to mature swell. Given the finite number of random frequency components, such simulations are valid only for a finite amount of time. Additionally, the lack of a dissipation range in the high frequency is expected to produce a slight overestimate of energy in this region of the spectrum; however, as will be shown later, this should not have a significant impact on the final results.

For this study, 200-s simulations were repeated within a Monte Carlo framework to create long sets of random waves using 50 sets of 50 random numbers to generate random 10000 -s wave records for each sea 
state simulated. In a simulation that neglects angular spreading, the height of the water surface at any time step is calculated from the linear approximation,

$$
\eta(t)=\sum_{i_{\mathrm{frq}}=1}^{N_{\mathrm{frq}}} z_{i_{\mathrm{frq}}},
$$

where $z_{i_{\mathrm{fr}}}$ is the individual contribution to the total surface height by each frequency component at a single point location in space and $i_{\text {frq }}$ is the frequency increment. For unidirectional simulations $z_{i_{\text {frq }}}$ is defined as

$$
z_{i_{\text {frq }}}=a_{i_{\text {frq }}} \cos \left(-\omega_{i_{\text {frq }}} t+\phi_{i_{\text {frq }}}\right) \delta \omega,
$$

where $\phi$ is the random phase of the linear waves assigned to each discrete frequency bounded in the range $[0,2 \pi]$, and $a$ is the amplitude of the wave at each frequency constituent.

An example of a 50-s surface record, shown in Fig. 2, was generated for a wave spectrum corresponding to 4-s spectral peak and $15 \mathrm{~m} \mathrm{~s}^{-1}$ wind speed. A zero-up crossing method was used to identify individual waves within the overall wave time series in these records which are marked in red circles in the figure. To inspect numerical aspects of our methodology, simulations were run with smaller time steps $(0.5$ and $0.25 \mathrm{~s})$, with more frequency constituents $\left(N_{\text {frq }}=391\right.$ and 781$)$, on a wider frequency range (up to $2 \mathrm{~Hz}$ instead of $1 \mathrm{~Hz}$ ) and with smaller bin widths. The impact of variations in bin width on the limiting breaking ratio had minimal effects that remained well under $10 \%$ for all tests. For this reason, we continued testing with the initial setup for simplicity and run-time efficiency.

Equation (2) contains a sufficient number of components to describe a realistic unidirectional random sea surface; however, the question of directional spreading must also be addressed. Directional spreading reduces the estimated orbital velocities in the mean direction, which affects the expected location of the transition frequency. Hence, additional simulations were executed with directional spectra to examine this impact of such directional spreading. Including directional constituents into $z_{i_{\text {frq }}}$ to run directional simulations results in

$$
\begin{aligned}
z_{\left(i_{\mathrm{frq}} j_{\mathrm{dir}}\right)}^{\prime}= & a_{i_{\mathrm{frq}}} \cos \left(k_{i_{\mathrm{frq}}} x_{i_{\mathrm{frq}}}-\omega_{i_{\mathrm{frq}}} t+\phi_{\left(i_{\mathrm{frq}}, j_{\mathrm{dir}}\right)}\right) \\
& \times \cos \left(\theta_{0}-\theta_{j_{\mathrm{dir}}}\right) \delta \omega \delta \theta \\
z_{i_{\mathrm{frr}}}= & \sum_{j_{\mathrm{dir}}=1}^{N_{\mathrm{dir}}} z_{\left(i_{\mathrm{frq}}, j_{\mathrm{dir}}\right)}^{\prime} .
\end{aligned}
$$

The difference from the unidirectional form is that the initial phase is specified for all frequencies and angles

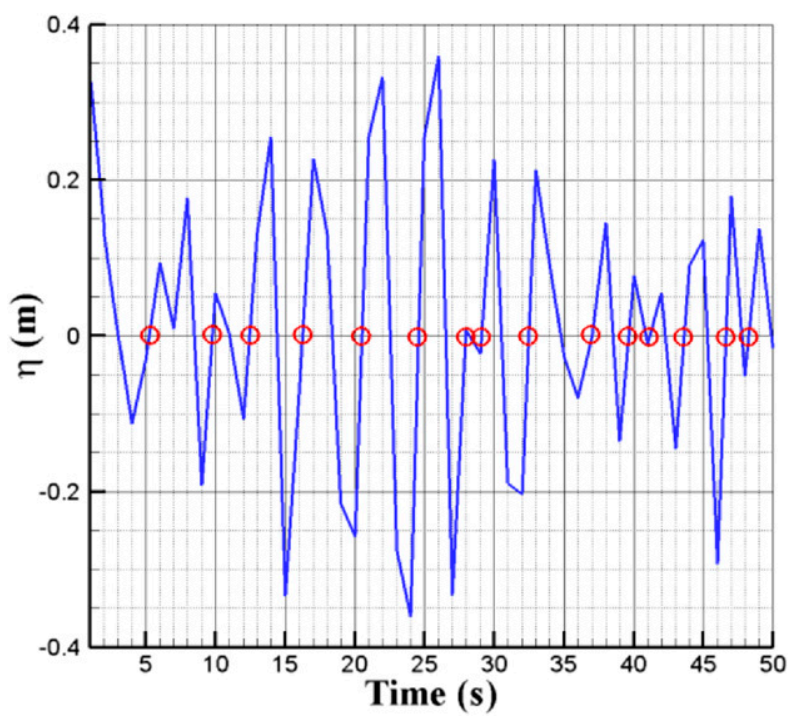

FIG. 2. The blue line represents the surface record, and the red circles are the zero-upcrossing points defining starting and ending times for each individual wave.

within the spectra. For these runs 15 angle bands were centered on the mean angle with $10^{\circ}$ increments which covered a total of $150^{\circ}$. For deep water $k_{i_{\mathrm{rrq}}}$ was simply found by using the dispersion relationship $\omega_{i_{\mathrm{frq}}}^{2}=g k_{i_{\mathrm{frq}}}$ and $x_{i_{\mathrm{frq}}}=C_{g_{i_{\mathrm{frq}}}} \cos (\theta) \Delta t$. Tests with directional (15) and frequency (191) constituents were executed with about 2800 components for these directional simulations.

In deep water, the horizontal orbital velocity $U_{\text {orb }}$ of narrow discrete bands is approximated by $a_{i_{\mathrm{frq}}} \omega_{i_{\mathrm{frq}}} \delta \omega$, neglecting nonlinear interactions, for unidirectional simulations. The partial sum up to the $i$ th frequency in unidirectional simulations produces orbital velocity of frequency components that are less than or equal to the orbital velocity of any prescribed upper limit $\omega_{i_{\text {frg }}}$, including the effects of random phases,

$$
U_{\text {orb }}\left(f_{i_{\mathrm{frq}}}, t\right)=\sum_{i=1}^{i_{\mathrm{frq}}} a_{i} \omega_{i} \cos \left(\varphi_{i}-\omega_{i} t\right) \delta \omega
$$

where $U_{\text {orb }}\left(f_{\text {ifra }}, t\right)$ is the estimated total orbital velocity produced by all the sum of phase-dependent velocity increment in a sea with random phases, with $\varphi_{i}$ defined as the initial random phase of the $i$ th frequency component at time $t$. For directional simulations, the directionally integrated form changes into

$$
\begin{aligned}
U_{\text {orb }}\left(f_{i_{\text {frq }}}, t\right)= & \sum_{i=1}^{i_{\text {frq }}} \sum_{j=1}^{N_{\text {ang }}} a_{i, j} \omega_{i, j} \cos \left(\varphi_{i, j}+k_{i} x_{i, j}-\omega_{i, j} t\right) \\
& \times \cos \left(\theta_{0}-\theta_{j}\right) \delta \omega \delta \theta,
\end{aligned}
$$


where $\varphi_{i, j}$ is the initial random phase of spectral component at the $i$ th frequency and $j$ th angle, with $N_{\text {ang }}$ representing the total number of angle components simulated within the spectrum.

The maximum orbital velocity for each upcrossing wave was calculated as a function of the upper limit of the sums in Eqs. (4) and (5), using zero-upcrossings to define individual waves and their maximum orbital velocities. This maximum value of $U_{\text {orb }}$ during the interval within this zero-crossing wave was defined as $\max \left[U_{\text {orb }}\left(f_{i_{\mathrm{rra}}, t},\right]\right.$ for each individual upcrossing wave. The ratio of this orbital velocity to the group velocity of the upcrossing wave, $C_{g}=g / 2 \omega$, represents the group velocity of the lowest frequency that is expected to break in this individual wave, defined as frequency $f_{b}$, given in deep water by

$$
f_{b}=\psi \frac{g}{\left(4 \pi U_{\text {orb }}\right)},
$$

where $\psi$ is the empirical coefficient established by the kinematic breaking criterion in various studies $(0.8-1.0)$. To test the sensitivity to the value of this coefficient, three different values of $\psi ; 0.7,0.8$, and 0.9 , were used in these simulations. An important distinction between $f_{b}$ and $f_{\mathrm{ti}}$ is that the former defines a single breaking event and varies probabilistically in time and space for a fixed spectrum, while the latter is the location where the rate of loss of energy to breaking matches the energy coming into that region of the spectrum. Significant spectral evolution occurs on time scales of $100-1000 \mathrm{~s}$ of these individual breaking events. In this study, 50 sets of breaking frequencies for each wave age and $\psi$ combinations were generated. Depending on the magnitude of $U_{\text {orb }}$ in Eq. (6), breaking could occur at higher frequencies, outside the modeled frequency range. The implication for very low wind speeds is that little or no white capping would be observed, consistent with Callaghan et al. (2008) who observed that the wind speed limit where little to no white capping was seen as approximately $5 \mathrm{~m} \mathrm{~s}^{-1}$.

These simulations yield a distribution of frequencies (probabilities) for breaking waves in each simulation. To estimate a single, expected value of $f_{\mathrm{ti}}$ requires an additional equation. In a detailed-balance form for source terms, such a balance would occur if the average value of $f_{\mathrm{ti}}$ produces a rate of energy loss equal to the rate of energy flux from the equilibrium range into the dissipation region $\Gamma_{E}^{+}$. As shown by Resio et al. (2001), such fluxes depend on the energy level within the equilibrium range, and can be estimated by

$$
\Gamma_{E}^{+}=\frac{\lambda \beta^{3}}{g}
$$

where $\beta$ is the reference wind velocity governing the momentum transfer rate into the wave field and $\lambda$ is a near-constant coefficient weakly related to angular spreading within the equilibrium region within a range of about 3-5. Given the type of angular distributions chosen in our work, we set $\lambda$ equal to 5 for our flux estimates.

Assuming that this breaking phenomenon primarily affects the spectral region, $f \geq f_{\text {ti }}$, the average rate of dissipative energy loss must balance the nonlinear energy fluxes to high frequencies due to nonlinear fluxes entering this region of the spectrum. In the context of a spectral estimate of energy densities within a turbulent cascade, the dimensional form of this region should be given by an $f^{-5}$ form, with directionally integrated form $E(f)=\alpha_{5} g^{2} f^{-5}$, with the total energy in a single breaking event given by

$$
\Delta E_{\mathrm{brk}_{m}}=\int_{f_{\mathrm{brk}_{m}}}^{\infty} \alpha_{5} g^{2} f^{-5} d f=\frac{\alpha_{5} g^{2} f_{\mathrm{brk}}^{-4}}{4} .
$$

Maximum orbital velocities in individual zero-crossing waves are random quantities. The probability distribution function (pdf) of breaking events from typical simulations is shown in Fig. 3, based on the breaking criterion used here for two different wave ages. Figure 4 shows the cumulative distribution function (CDF) of the rate of energy removed by such breaking over a simulation, compared to the energy flux for an equilibrium with a $5 \mathrm{~m} \mathrm{~s}^{-1}$ wind speed and a spectral peak frequency of $0.25 \mathrm{~Hz}$ and is typical of the simulated distributions.

As can be seen in Fig. 4, the CDF of energy losses increases somewhat exponentially in the vicinity of the point where it is intersected by the dashed line. Such behavior supports the interpretation that the mean value of energy loss should provide a stable estimate of the balance point between nonlinear fluxes and breaking over many wave periods. Any deviation will create a substantial imbalance since the rate of change of the total energy loss is very dependent on variations in the breaking frequency, a small variation in this frequency will be associated with a relatively large change in the rate of energy loss. Thus, the variation in cumulative energy losses is highly dependent on the breaking frequency and is a reasonable first approximation for the point of balance on a time scale of many wave breaking events as used in spectral models. 

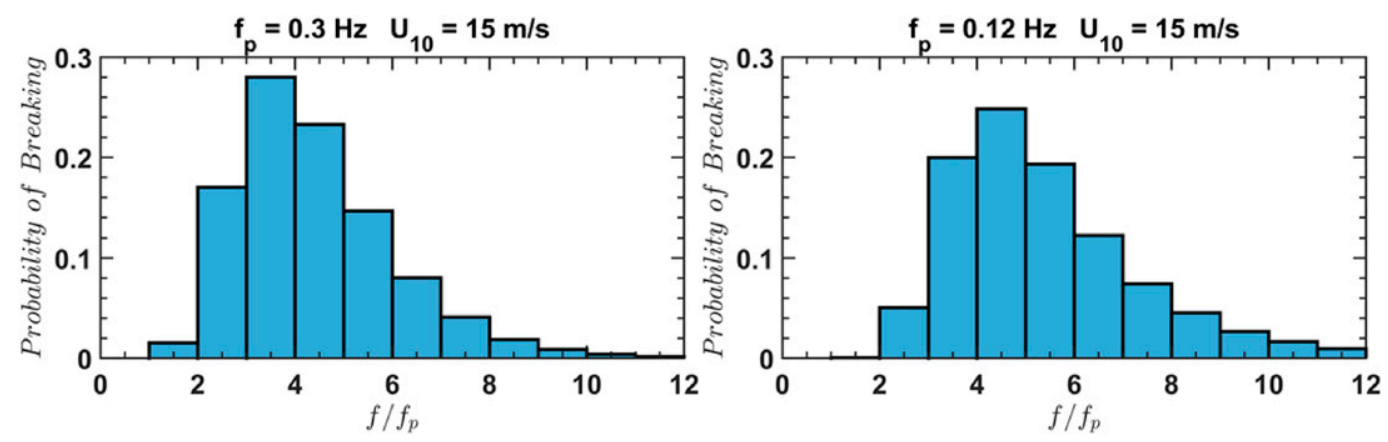

FIG. 3. Probability distribution functions for two different simulations under two different conditions, one with a (left) lower wave age and (right) higher wave age. The case for lower wave age breaking probabilities closer to the peak are higher.

Using this approximation, we obtain the final form for our energy balance at the breaking frequency and nonlinear fluxes:

$$
\frac{\Delta E_{b}}{\Delta t}=\frac{1}{t_{\text {tot }}}\left\langle\Delta E_{b_{m}}\right\rangle \approx \bar{f}_{b} \alpha_{5} g^{2} \frac{\sum_{m=1}^{N_{\text {tot }}} \bar{f}_{b_{m}}^{-4}}{4 N_{\text {tot }}}=\Gamma_{E}^{+},
$$

where $\Delta E_{b} / \Delta t$ is the energy lost per unit time, $t_{\text {tot }}$ is the total time simulated, $N_{\text {tot }}$ is the total number of breaking waves over the simulation time, and $\bar{f}_{b}$ is the mean breaking rate, with the subscript $m$ denoting the $m$ th breaking event in the simulation and the ensemble bracket $\langle\ldots\rangle$ denotes an averaging operator.

Equation (9) provides link between the nonlinear flux rate and the mean breaking frequency that matches the fluxes past this frequency. This can also be used to represent the frequency at which there is a transition from an equilibrium-range form governed by nonlinear fluxes to a spectral range dominated by breaking. This transition from an $f^{-4}$ spectral form to an $f^{-5}$ spectral form as has been observed in many studies (Birch and Ewing 1986; Resio et al. 2004; Long and Resio 2007). At transition frequency $f_{\text {ti }}$

$$
\left(\frac{\Delta E_{b}}{\Delta t}\right)_{f_{\mathrm{ti}}}=\Gamma_{E}^{+},
$$

where $f_{\mathrm{ti}}$ should be located at the point where the rate of nonlinear energy fluxes into higher frequencies balances the mean rate of energy loss due to random breaking events, assuming negligible wind input into this whitecapping region. This produces a Phillips $f^{-5}$ spectral form at frequencies above $f_{\mathrm{ti}}$ in which sporadic breaking maintains an overall balance with the incoming energy fluxes and forms an energy cascade, similar to that found in other turbulence. A major difference from the initial breaking assumption by Phillips is that the empirical coefficient $\left(\alpha_{5}\right)$ in Eq. (8) is a constant in his hypothesized form, while the value of this coefficient in our formulation must vary to match the value of the equilibrium range $f^{-4}$ spectral form at the transition point $f_{\text {ti }}$. Energy transferred into the breaking region by nonlinear fluxes should be lost in an average sense by the dissipation within the breaking zone to current generation and turbulence.

Observational evidence (Long and Resio 2007; Romero and Melville 2010; Lenain and Melville 2017) implies an inverse relationship between the wave age and the location of the transition zone. For instance, for swell, the transition to the breaking region is expected to occur at much higher frequencies relative to the spectra peak frequency compared to that found in young spectra. Our assumption is that wave breaking begins to play a very strong role in the overall energy balance at this frequency and the spectral form transitions from the $f^{-4}$ to an $f^{-5}$ spectral form. Denoting the energy

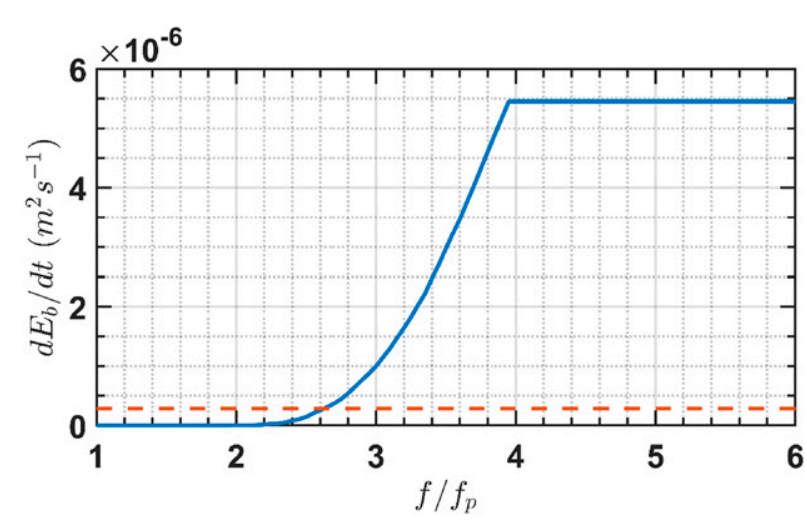

FIG. 4. Positive energy fluxes (red dashed line) (originated from the peak frequency) found by using Eq. (7) drawn against the breaking energy rate (blue solid line) for a simulation initiated with a peak frequency of $0.25 \mathrm{~Hz}$ and wind speed of $5 \mathrm{~m} \mathrm{~s}^{-1}$. They intersect around $2.5 f / f_{p}$, which denotes the transition frequency under this particular condition. 


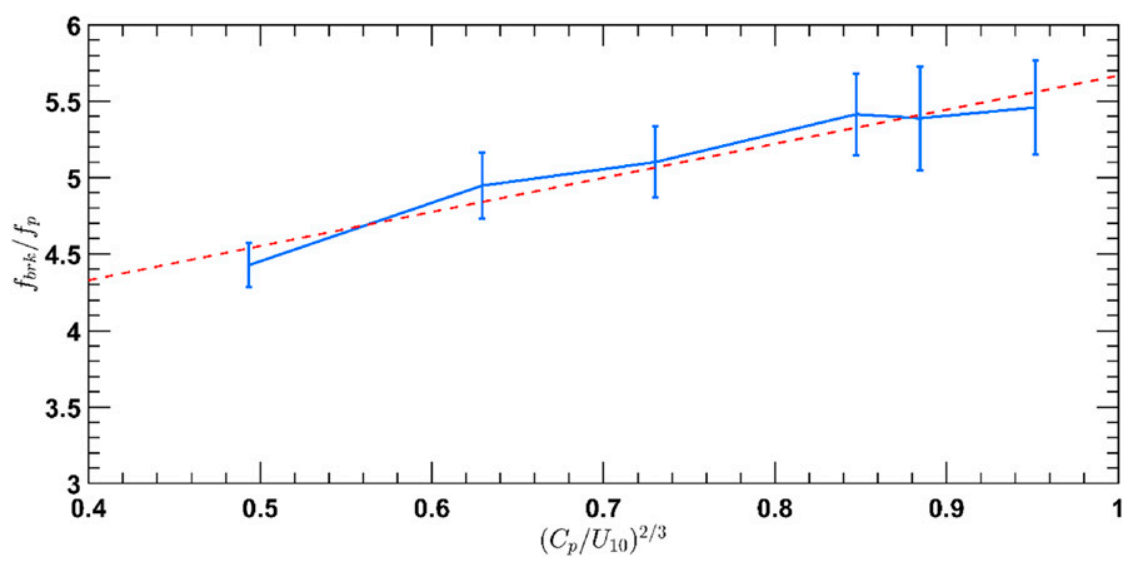

FIG. 5. The $f_{\text {brk }} / f_{p}$ plotted against $\left(C_{p} / U_{10}\right)^{2 / 3}$ to examine the proposed linear form in Eq. (13) for six different wave age values. Blue dots are the mean results for each random set given with $95 \%$ confidence limits (blue line). The linear fit is shown as the red dashed line.

coefficient as $\alpha_{5}$ for the $f^{-5}$ spectra, constrained by Eq. (9), the balance between the breaking range and the equilibrium range should lie close the point defined in Eq. (8) as $\bar{f}_{b}$. Neglecting dissipation less than $\bar{f}_{b}$, the directionally integrated energy into frequencies above $\bar{f}_{b}$ and out of the spectral region above this frequency must match, that is,

$$
\Gamma\left(f_{\mathrm{fti}}\right)=\alpha_{4} \beta g f_{\mathrm{ti}}^{-4}=\alpha_{5} g^{2} f_{\mathrm{ti}}^{-5}=\frac{\left\langle\Delta E_{\mathrm{brk}}\right\rangle}{\Delta t} .
$$

Combining Eqs. (7) and (9), we obtain the relationship

$$
\frac{\lambda \beta^{3}}{g}=\frac{\alpha_{5}}{4} g^{2} \bar{f}_{b}^{-3} .
$$

Combining Eq. (12) with Eq. (11) yields

$$
\lambda \beta^{2}=\frac{\alpha_{4}}{4} g^{2} \bar{f}_{b}^{-2} .
$$

Grouping constants and simplifying again, we obtain the relationship

$$
\chi \beta=\frac{g}{\bar{f}_{\text {brk }}},
$$

where $\chi$ is a coefficient that represents dimensionless constants derived from observations combined with the assumed value for the flux constant.

Resio et al. (2004) presented observational evidence that $\beta \approx\left(U_{10}^{2} C_{p}\right)^{1 / 3}$, which when combined with $C_{p}=g / \omega_{p}$ and Eq. (14) yields the relationship

$$
\chi \frac{\bar{f}_{b}}{f_{p}} \sim\left(\frac{C_{p}}{U_{10}}\right)^{2 / 3} .
$$

This implies a relationship between inverse wave age and the breaking location in which $\chi$ is a dimensionless coefficient that systematically shifts location of the transition point with respect to the location of the spectral peak. It also makes it clear that $f_{\mathrm{ti}} \approx \bar{f}_{b}$, as must be the case for these two terms as used in this paper.

\section{Results}

To validate Eq. (15), simulations were initiated using 50 sets of 50 random seed values for 6 different wave ages from young waves to swell cases. In Fig. 5, the abscissa of the plot shows the wave age factor as given in Eq. (15). The ordinate displays the mean of the $f_{\mathrm{ti}} / f_{p}$ values that were calculated for each random set and the error bars show the $95 \%$ confidence interval, based on the standard deviation and the number of cases simulated. The linear fit added here indicates that Eq. (15) provides a very reasonable approximation of relationship between wave age and the location of the dimensionless transition frequency, $\hat{f}_{\mathrm{ti}}=\bar{f}_{\mathrm{ti}} / f_{p}$. For high wind speeds (lower wave age) the uncertainty (error bars) of $f_{\text {brk }} / f_{p}$ is smaller as it can be seen from the plot, suggesting that, as the waves continue to develop and the transition frequency shifts to higher frequencies, the estimated location of $\hat{f}_{\mathrm{fti}}$ becomes more variable around its mean value. Since we have neglected the effect of spectral peakedness in this analysis, this behavior might be expected when wave spectra are so young that the location of the transition frequency approaches the spectral peak frequency.

One of the main tenets of this work is that the predicted energy loss rate is related to the magnitude of the positive energy fluxes calculated from Eq. (7). The amount of energy lost to breaking in the dissipation zone 


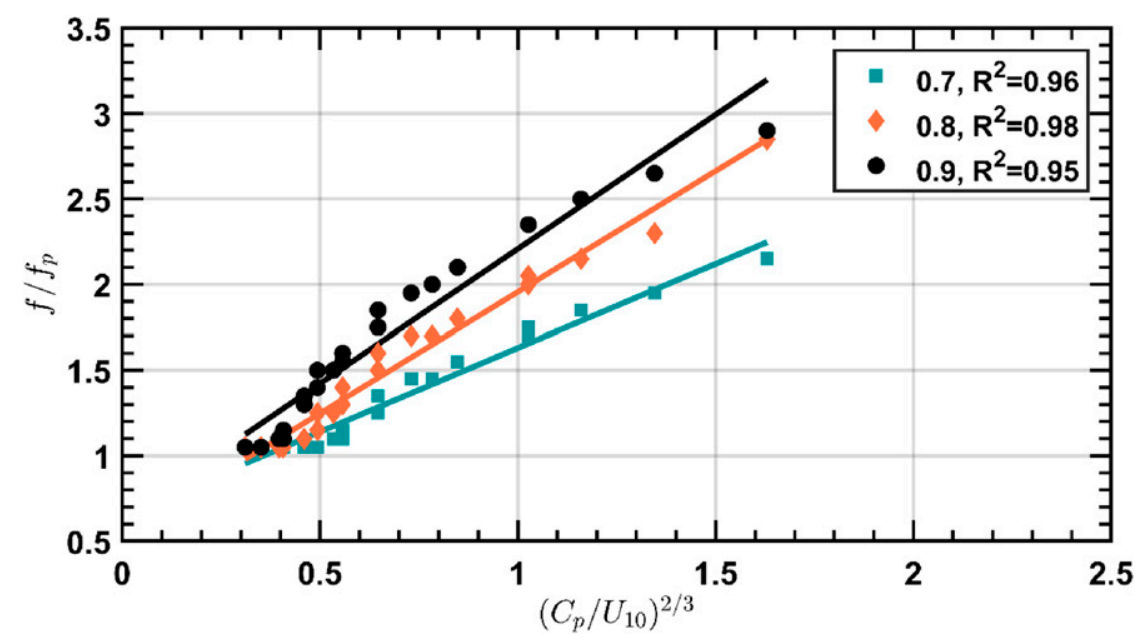

FIG. 6. Normalized transition frequency vs wave age parameter here are shown for 72 different simulations, where each dot represents a simulation. Three different colored lines show linear fits for $0.7,0.8$, and 0.9 breaking rates and the legend shows the $R^{2}$ values for each line.

for any breaking event is estimated using Eq. (10). Over the total duration of the simulations for various initial spectra, breaking may occur at any of the frequency points due to its randomness, as shown in Fig. 3. However, the rate of energy loss is expected to depend on the recurrence of breaking and the amount dissipated energy of a particular frequency constituent. For lower transition frequencies, the energy loss rate to breaking is high, indicating that the magnitude of positive energy fluxes is large. Our basic assumption regarding the breaking source term is

$$
\frac{\partial E_{b}}{\partial t}=\iint S_{\text {brk }}(f, \theta) d f d \theta .
$$

The simulations were executed for $500000 \mathrm{~s}$ in unidirectional tests and $250000 \mathrm{~s}$ in the directional tests. Figure 4 showed the positive flux rate originating from the peak (red dashed line) found by using Eq. (7) compared to the estimated rate of energy loss (blue line) for a simulation initiated with a peak frequency of $0.25 \mathrm{~Hz}$ (the upper frequency limit for this particular simulation was $1 \mathrm{~Hz}$ ), a wind speed of $5 \mathrm{~m} \mathrm{~s}^{-1}$. The value of $\psi$ for this run is 0.8 . Their intersection is approximately at $2.5 f / f_{p}$, which implies that wave spectra with this inverse wave age would transition from an $f^{-4}$ to an $f^{-5}$ near a frequency of $2.5 f / f_{p}$ under the assumptions introduced here. The estimated energy flux contains no empirical factors in this figure beyond the assumed value of $\psi$, which is consistent with the data presented in Resio et al. (2001).

Figure 4 represents a single peak frequency and wind speed condition. To cover a wider range of the sea states, 24 different conditions from very young waves to well-developed waves were generated using peak frequencies of $0.15,0.2,0.25$, and $0.3 \mathrm{~Hz}$ and wind speeds of $5,10,15,20,25$, and $30 \mathrm{~m} \mathrm{~s}^{-1}$. These simulations were initiated with 50 sets of 50 random seed values over a total duration of $500000 \mathrm{~s}$. The intersection points between the positive energy fluxes and the energy loss rates were found using the same methodology as introduced here for each case. To investigate the influence of the empirical coefficient of the kinematic criterion in Eq. (6) these tests were repeated for $\psi$ values of $0.7,0.8$, and 0.9 , yielding a total sample size with 72 points. Subsequently, these points were plotted against the estimate rates as a function of $\left(C_{p} / U_{10}\right)^{2 / 3}$ to obtain Fig. 6 . This figure also contains linear fits for each of the empirical coefficients and their $R^{2}$ values.

As shown in Fig. 6, the agreement between the two rates and the associated linear correlation values $\left(R^{2}\right)$ is high. An examination of this plot shows that for young waves the dissipation zone reaches to the peak, and the equilibrium range is essentially nonexistent under these circumstances. On the other hand, when the wind speed is low the transition frequency $f_{\mathrm{ti}}$ shifts toward higher frequencies. For $\psi=0.8$, the linear relationship for the dimensionless transition frequency is given as

$$
\hat{f}_{\mathrm{ti}}=\frac{\bar{f}_{b}}{f_{p}}=1.4\left(\frac{C_{p}}{U_{10}}\right)^{2 / 3} .
$$

with a random deviation of 0.05 around the mean function; however, some patterns of deviation are apparent in the overall behavior, which deserve additional study. It is likely that a more detailed additional study is required to investigate dimensionless transition 


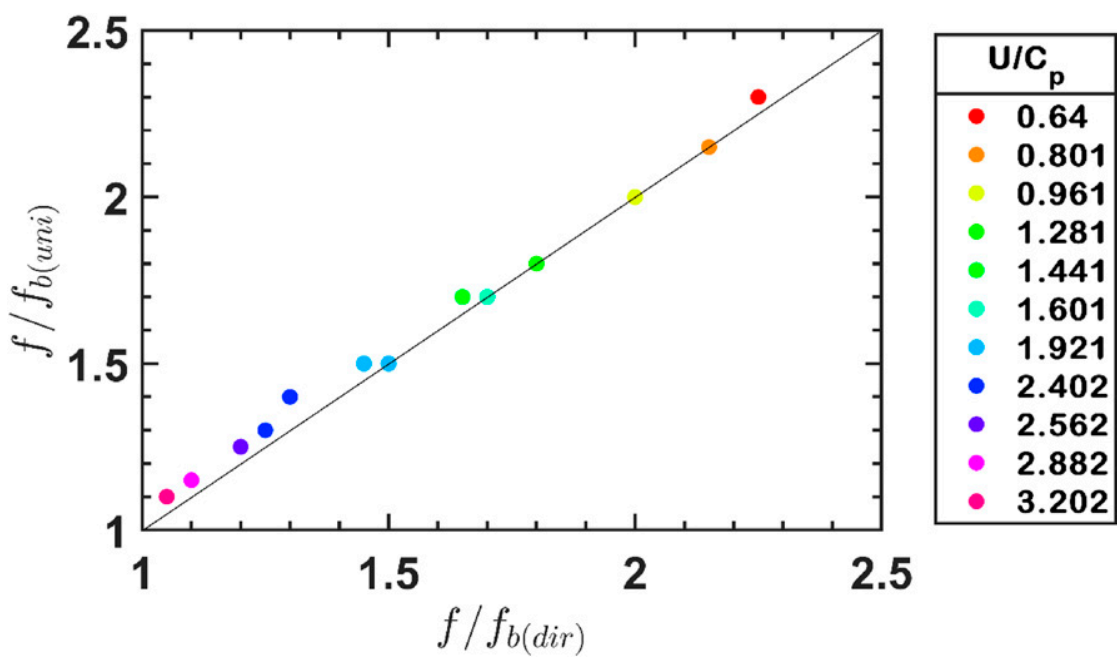

FIG. 7. Comparison of $f / f_{\text {brk }}$ results from unidirectional and directional tests over a range of inverse wave age conditions.

frequency in the region $\hat{f}_{\mathrm{ti}} \leq 1.6$, since in this region the source term for nonlinear interactions begins to be strongly affected by the sharp cutoff in spectral energy on the low-frequency side of the spectral peak.

For completeness of our theory we compared results from unidirectional and directional tests for varying wave ages. Here, we initiated directional runs with $\psi$ rate of 0.8 over a total duration of 250000 s, completed the same analysis as explained above, and on Fig. 7, we compared locations of transition frequencies for a wide range of sea states. As it is apparent from the figure, for all of these wind-sea conditions the difference is relatively small due to the strong dependence of the breaking dissipation rate on the location of $\hat{f}_{\text {ti }}$.

These figures support the linear relationship given in Eq. (15) between the two parameters. A comparison of this hypothesized relationship to field data by Long and Resio (2007) is shown in Fig. 8, where the compensated and normalized energy spectra were grouped by their inverse wave ages to indicate transition frequencies with black dots. Since the range of young waves in Fig. 8, is limited to the range for $1.6<\hat{f}_{\mathrm{ti}}<3.4$. Overall, comparison between Figs. 6 and 8 shows good agreement between observed and predicted transition frequencies and Table 1 gives the relationship between the transition frequencies and the inverse wave age based on these simulations.

\section{Discussion and conclusions}

A kinematic criterion for wave breaking is used here as a limiter for individual wave breaking in a wave field. It is shown that this form of breaking can be converted into an average dissipation rate for application within a spectral context using Monte Carlo simulations. Two important constraints are maintained in these simulations: 1) the spectral energy at the point of transition represents the point where the total energy loss due to

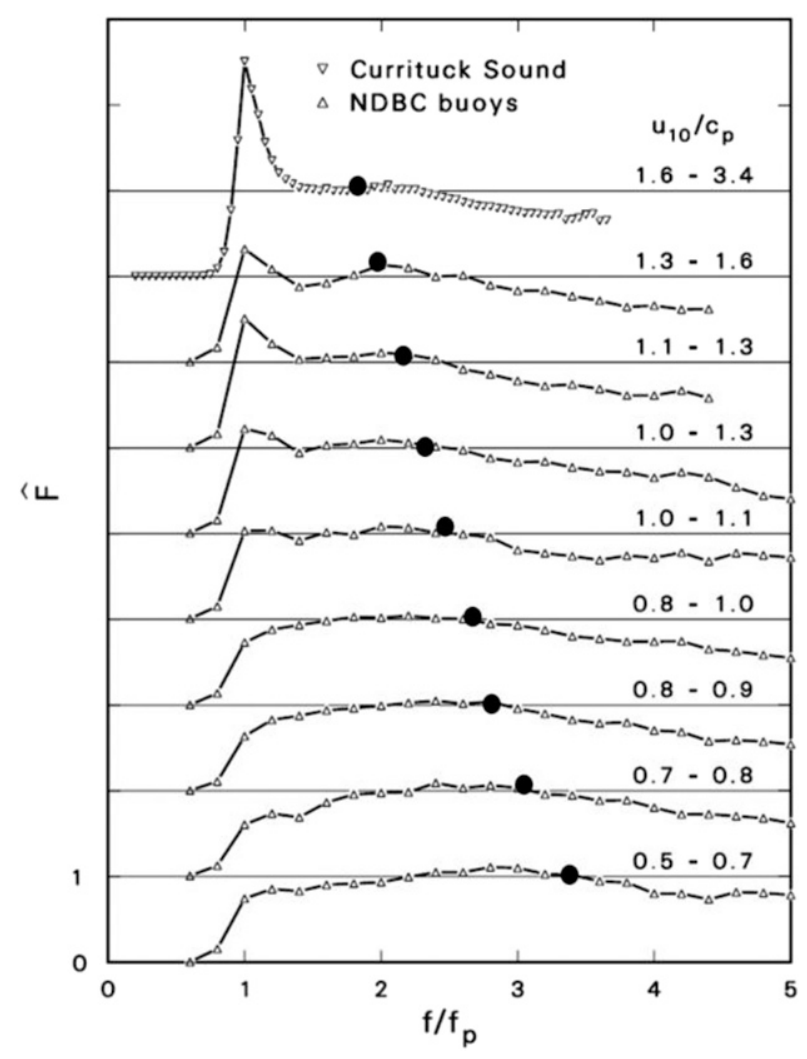

FIG. 8. Compensated and normalized energy densities grouped and averaged according to their inverse wave ages (Long and Resio 2007). Transition frequencies are marked with the black dot. 
TABLE 1. Transition frequencies defined by the inverse wave age values.

\begin{tabular}{lc}
\hline \hline Inverse wave age $\left(U / c_{p}\right)$ & $\begin{array}{c}\text { Nondimensional transition } \\
\text { frequency }\left(f_{\text {ti }}\right)\end{array}$ \\
\hline 0.7 and lower & 2.9 \\
0.8 & 2.7 \\
1 & 2.5 \\
1.2 & 2.2 \\
1.6 & 2 \\
2 and higher & 1.5 \\
\hline
\end{tabular}

sporadic breaking matches the nonlinear flux of energy into the zone and 2) the energy densities of the equilibrium range form and the dissipation zone must also match at this point.

The point of transition is defined as the frequency at which the ratio of the group velocity associated with that frequency divided by the cumulative horizontal orbital velocity estimated from the sum of orbital velocities produced by lower frequencies exceeds 0.85 . All higher frequencies also surpass the limiting value for breaking, so each exceedance leads to a single breaking event in the wave field that removes energy from all frequencies higher than this threshold. The value of $f_{b}$ also provides needed information needed to estimate the overall dissipation rate due to breaking. The kinematic breaking criterion used here is supported by several field and laboratory studies (Waseda et al. 2014; Saket et al. 2017; Shemer and Liberzon 2014), and is consistent with numerical studies of focused wave phases, for the special case when breaking occurs at the spectral peak (Barthelemy et al. 2018; Derakhti and Kirby 2016). Essentially all of these papers show a clear association between wave breaking and the ratio of horizontal orbital velocities to a characteristic wave velocity.

It should be noted that this breaking mechanism is functionally similar to the criterion for "dominant" wave breaking developed by Barthelemy et al. (2018). In the Barthelemy et al. laboratory experiment, wave focusing is specifically developed such that strong convergence occurs in the spectral peak region. In the Monte Carlo simulations here, we see that such singlevalued convergence is not very likely in random-phase wave spectra, since they depend on statistical properties which must be approximated by a large number of spectral frequency-angle components. In fact, none of our cases developed wave breaking at frequencies less than $1.2 f_{p}$ in the spectra peak region for typical spectral shapes with wave ages characteristic of open ocean conditions [inverse wave ages $\left(u_{10} / c_{p}\right)<1.8$ ]. This does not mean that is does not happen in very young waves, since we see cases of such spectra in both the Birch and Ewing (1986) data and the Long and Resio (2007) data.
It should also be clear that the statistical distribution around the mean value certainly can extend to a point where some breaking might be at the spectral peak; however, it is unlikely that the mean value of breaking will extend that far toward low frequencies.

Typical analyses of wave spectra, even at short fetches, do not separate spectra into different wave age bins for analysis and many of the measurement sites used for careful spectral shape analyses are located in relatively shallow water; however, a careful study by Birch and Ewing (1986) provides additional valuable insight into the existence of two different equilibrium range forms that are quantified here. Figure 9, based on their measurements in a small reservoir, found very young waves (high wind speeds) exhibited $f^{-5}$ behavior throughout the typical range of frequencies considered to fall within the equilibrium range. They also found spectra at lower wind speeds that exhibited $f^{-4}$ behavior over a significant portion of the equilibrium range before transitioning to an $f^{-5}$ form. Figure 10 shows examples of these spectra used to demonstrate this behavior in their report.

In the context of observational support for this form or wave breaking, Zhao and Toba (2001) found that a very good fit to whitecap coverage across a broad range of scales was given by a parameter proportional to the product of friction velocity cubed times wave age, where wave age is defined as the peak phase speed divided by the wind speed, that is, whitecap coverage is proportional to $u_{*}^{2} c_{p}$, where $u_{*}$ is friction velocity and $c_{p}$ is the phase velocity of the spectral peak. This relationship is consistent with the form of equilibrium ranges based on nonlinear fluxes found in Resio et al. (2004).

Today, most operational wave models still use a parametrically determined frequency value to transition to a parametric $f^{-5}$ frequency range and use a prescriptive spectral form at frequencies higher than this value. This implies that $\alpha_{5}$ is a universal constant. In the physical context of breaking and other source terms presented here, arguments presented in Ardag and Resio (2019) suggest strongly that wind-wave interactions in the equilibrium range contains zero net wind input unless it is balanced by dissipation. As shown there any net source term will force the spectrum away from an $f^{-4}$ form. In turn, this suggests that the dissipation $\left(f^{-5}\right)$ range likely is the source of all apparent roughness in wind-wave interactions; however, following up on this topic is beyond the scope of this paper.

In terms of alternative developments that include dissipation, a new source term incorporated into some recent source term packages considers the exceedance probability related to an integrated wave steepness factor. The final version of $S_{\mathrm{ds}}$ is given by (Babanin et al. 2010) 

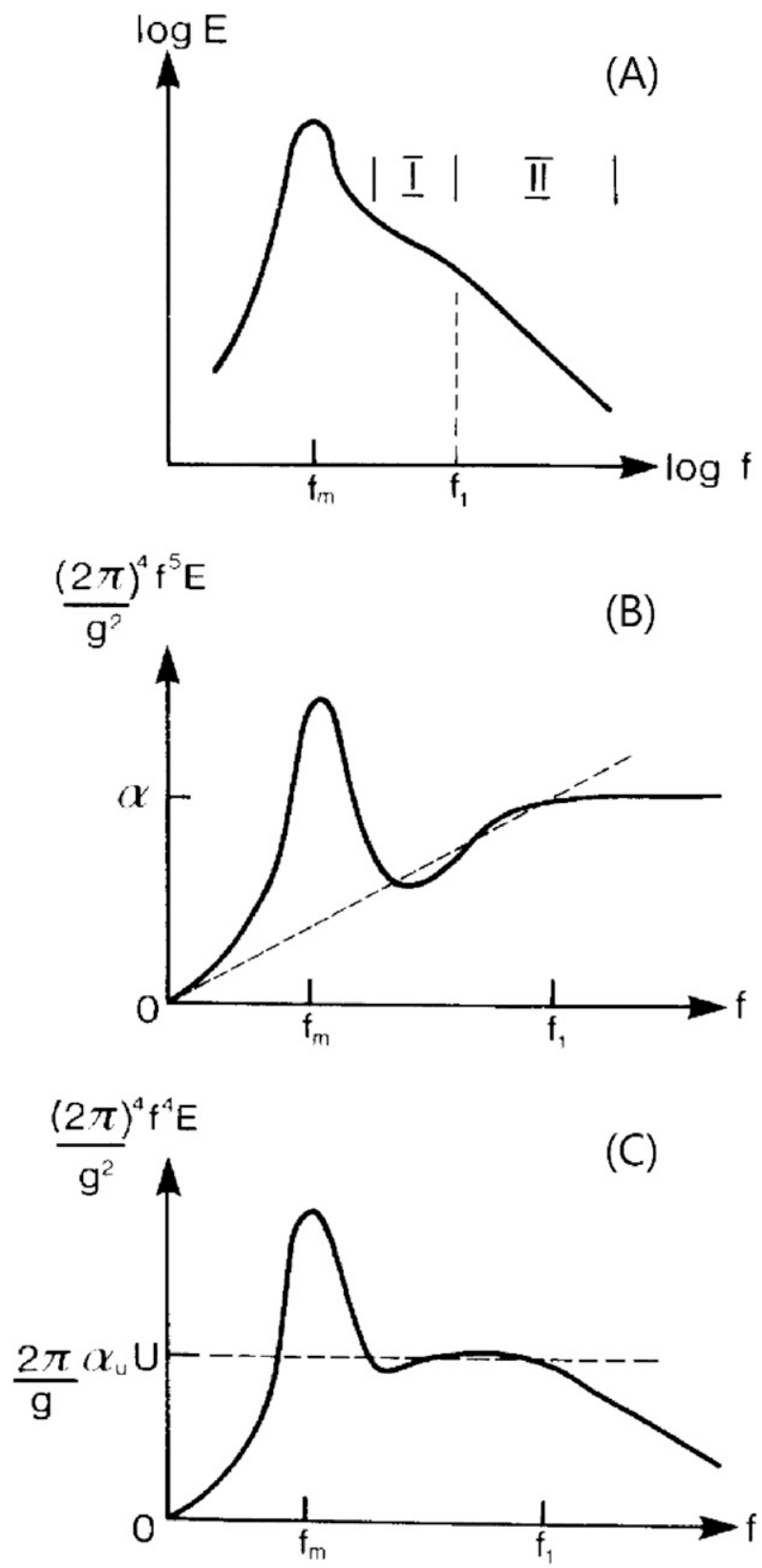

FIG. 9. (a) An example spectrum from Birch and Ewing's (1986) data is given. (b) The same spectrum is multiplied by $f^{5}$ to show the $f^{-5}$ zone. (c) The same approach, but this time the same spectrum is multiplied by $f^{4}$ to show the equilibrium zone. Here $f_{1}$ indicates the transition frequency ( $f_{\mathrm{ti}}$ in our notation).

$$
\int S_{\text {in }}(f) d f=R \int S_{\mathrm{ds}}(f) d f
$$

where $R$ is an empirical constant defined by Donelan (1998). This breaking form implies a detailed balance exists between two processes, wind input and wave breaking, that occur on very different time scales, which then implies that these processes operate over the same
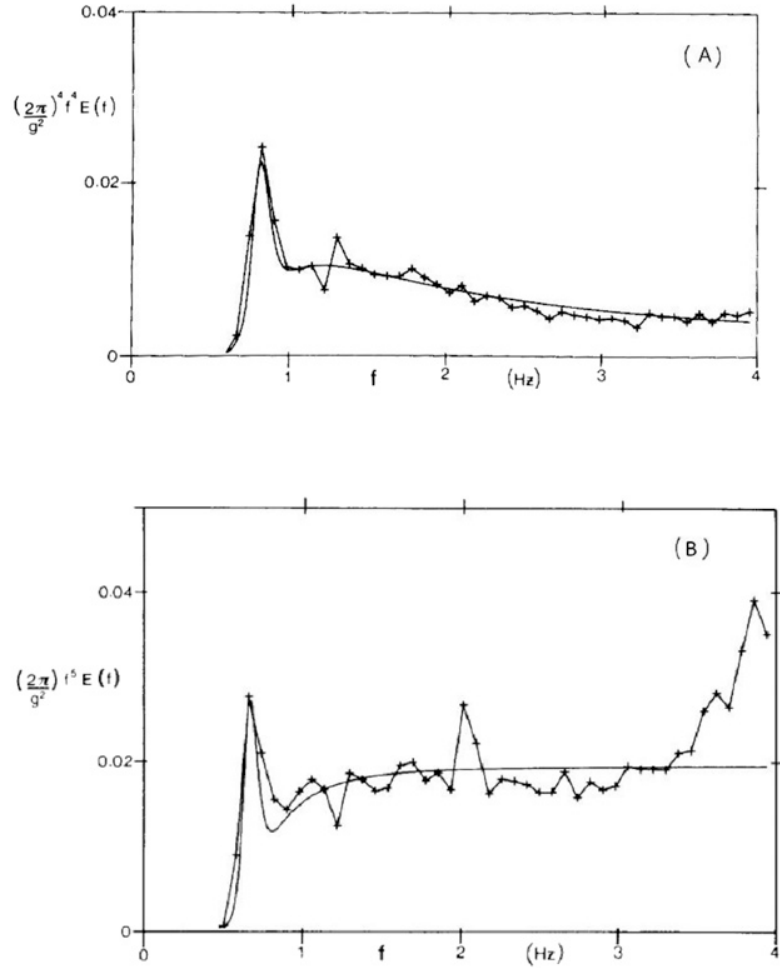

FIG. 10. Two plots taken from Birch and Ewing (1986) for wind speeds of (a) 6.7 and (b) $10.8 \mathrm{~m} \mathrm{~s}^{-1}$. In both plots there is a short equilibrium range followed by the dissipation range due to the high inverse wave age conditions.

range of frequencies and directions. This form also would not explain the observed transition to a dissipative range in the spectrum. As is shown in this paper, the transition frequency $f_{\mathrm{ti}}$ occurs at a variable location relative to the peak frequency. Also, the neglect of $S_{\text {nl }}$ indicates that Eq. (18) is based on a parametric rather than a detailed-balance relationship. Given that detailedbalance wave models require orders of magnitude more computer time than second-generation, parametric source-term models, it seems that such assumptions are more appropriate for second-generation models.

Beside the data introduced in this paper that seems to support the existence of a quantitative limit to the ratio of the orbital velocity at the surface to the wave propagation velocity, some existing data from previous studies appear to offer some support for this interpretation. A set of experiments involving the superposition of swell waves onto characteristic local sea spectra (Smith 2003) showed that mechanically generated wave spectra superposed onto wind-generated spectra reduced energy levels in the local sea portion of the spectrum, consistent with the expected increase in wave breaking in higher frequency waves due to the addition of the swell orbital velocities. 
The theoretical perspective developed in this paper also raises an interesting question regarding the use of empirical estimates of a power-law behavior for the slope of the equilibrium range, suggesting that such efforts may actually be blending two different regions of the spectrum into the same analysis, leading to estimate power laws of the form $f^{-n}$ result in values of $n$ that fall between 4 and 5. Thus, applying a logarithmic fit to a fixed range of frequencies, without grouping it according to the wave age, might be misleading (Rodríguez and Soares 1999; Umesh et al. 2018).

The main findings of this work are as follows:

1) Wave fields produced by random-phase simulations show that waves in the range of frequencies above the spectral peak will surpass the kinematic limit for wave breaking at a far greater rate than waves in the vicinity of the spectral peak, consistent with the detailed modeling by Irisov and Voronovich (2011), who showed the dominant breaking began at a midrange of frequencies rather than at the spectral peak or only very high frequencies.

2) The transition frequency from a nonlinear energyflux spectral form $\left(f^{-4}\right)$ to a dissipative spectral region $\left(f^{-5}\right)$ is found to be consistent with the location where the mean rate of breaking (determined by Monte Carlo simulations using the kinematic breaking limit) matches the energy flux toward higher frequencies passing through this point of the spectrum.

3) Under the assumptions made here, the estimated transition frequency $f_{\mathrm{ti}}$ is found to be a function of inverse wave age $\left(C_{p} / U_{10}\right)^{2 / 3}$ as shown in Eq. (15).

4) The relationship between the transition frequencies derived here is shown to be consistent with observations (Long and Resio 2007) as shown in Table 1. This allows the new breaking term to be adapted for use in existing operational wave models as a predictor of the transition frequency and as an aid to the estimation of whitecap coverage. This is an important step toward providing a physics-based source term that enhances the stability of simulations using the nonlinear transfer source term. Since this region of the spectrum is typically where stability problems in this source term are most pervasive, the incorporation of this transition into these models should enable the use of enhanced (longer) time steps and improved estimates of wind-wave momentum exchanges related to spectral shape.

Acknowledgments. A portion of this work conducted by Dr. Ardag and Dr. Resio was supported by the
National Ocean Partnership Program (NOPP), Award Number N00014-12-1-0127. The views expressed here do not necessarily reflect the views of this organization. The authors thank anonymous reviewers for their extremely valuable comments as they clarified and enhanced the manuscript and for their encouragement regarding its relevance.

\section{REFERENCES}

Alves, J. H. G. M., and M. L. Banner, 2003: Performance of a saturation-based dissipation-rate source term in modeling the fetch-limited evolution of wind waves. J. Phys. Oceanogr., 33, 1274-1298, https://doi.org/10.1175/1520-0485(2003)033<1274: POASDS $>2.0 . \mathrm{CO} ; 2$.

Ardag, D., and D. T. Resio, 2019: Inconsistent spectral evolution in operational wave models due to inaccurate specification of nonlinear interactions. J. Phys. Oceanogr., 49, 705-722, https:// doi.org/10.1175/JPO-D-17-0162.1.

Babanin, A. V., I. R. Young, and M. L. Banner, 2001: Breaking probabilities for dominant surface waves on water of finite constant depth. J. Geophys. Res., 106, 11 659-11676, https:// doi.org/10.1029/2000JC000215.

— K. K. N. Tsagareli, I. R. Young, and D. J. Walker, 2010: Numerical investigation of spectral evolution of wind waves. Part II: Dissipation term and evolution tests. J. Phys. Oceanogr., 40, 667-683, https://doi.org/10.1175/2009JPO4370.1.

Banner, M. L., and X. Tian, 1998: On the determination of the onset of breaking for modulating surface gravity water waves. J. Fluid Mech., 367, 107-137, https://doi.org/10.1017/ S0022112098001517.

_ for two-dimensional deep-water wave groups. J. Fluid Mech., 585, 93-115, https://doi.org/10.1017/S0022112007006568.

_ A. V. Babanin, and I. R. Young, 2000: Breaking probability for dominant waves on the sea surface. J. Phys. Oceanogr., 30, 3145-3160, https://doi.org/10.1175/1520-0485(2000)030<3145: $\mathrm{BPFDWO}>2.0 . \mathrm{CO} ; 2$.

Barthelemy, X., M. L. Banner, W. L. Peirson, F. Fedele, M. Allis, and F. Dias, 2018: On a unified breaking onset threshold for gravity waves in deep and intermediate depth water. J. Fluid Mech., 841, 463-488, https://doi.org/10.1017/ jfm.2018.93.

Birch, K. G., and J. A. Ewing, 1986: Observations of Wind Waves on a Reservoir. Institute of Oceanographic Sciences Rep. 234, $37 \mathrm{pp}$.

Callaghan, A., G. de Leeuw, L. Cohen, and C. D. O'Dowd, 2008: Relationship of oceanic whitecap coverage to wind speed and wind history. Geophys. Res. Lett., 35, L23609, https://doi.org/ 10.1029/2008GL036165.

Derakhti, M., and J. T. Kirby, 2016: Breaking-onset, energy and momentum flux in unsteady focused wave packets. J. Fluid Mech., 790, 553-581, https://doi.org/10.1017/ jfm.2016.17.

__, M. L. Banner, and J. T. Kirby, 2018: Predicting the breaking strength of gravity water waves in deep and intermediate depth. J. Fluid Mech., 848, R2, https://doi.org/ 10.1017/jfm.2018.352.

Donelan, M. A., 1998: Air-water exchange processes. Physical Processes in Lakes and Oceans, J. Imberger, Ed., Coastal and Estuarine Studies, Vol. 54, Amer. Geophys. Union, 19-36. 
_- 2001: A nonlinear dissipation function due to wave breaking. Ocean Wave Forecasting Conf., Shinfield Park, Reading, ECMWF, 87-94, https://www.ecmwf.int/node/9094.

— _. J. Hamilton, and W. H. Hui, 1985: Directional spectra of wind-generated waves. Philos. Trans. Roy. Soc. London, 315A, 509-562, https://doi.org/10.1098/rsta.1985.0054.

Duncan, J. H., 1981: An experimental investigation of breaking waves produced by a towed hydrofoil. Proc. Roy. Soc. London, 377A, 331-348, https://doi.org/10.1098/rspa.1981.0127.

Elgar, S., 2007: Waves in oceanic and coastal waters. Oceanography, 20, 133-135, https://doi.org/10.5670/oceanog.2007.42.

Forristall, G. Z., 1981: Measurements of a saturated range in ocean wave spectra. J. Geophys. Res., 86, 8075-8084, https://doi.org/ 10.1029/JC086iC09p08075.

Gerstner, F., 1809: Theorie der Wellen. Ann. Phys., 32, 412-445, https://doi.org/10.1002/andp.18090320808.

Hasselmann, K., 1961: On the non-linear energy transfer in a wave spectrum. Ocean Wave Spectra, Prentice Hall, 191-197.

_ 1974: On the spectral dissipation of ocean waves due to white capping. Bound.-Layer Meteor., 6, 107-127, https://doi.org/ 10.1007/BF00232479.

_ , and Coauthors, 1973: Measurements of wind-wave growth and swell decay during the Joint North Sea Wave Project (JONSWAP). Ergnzungsh. Dtsch. Hydrogr. Z., A8, 95 pp.

Irisov, V., and A. Voronovich, 2011: Numerical simulation of wave breaking. J. Phys. Oceanogr., 41, 346-364, https://doi.org/ 10.1175/2010JPO4442.1.

Itay, U., and D. Liberzon, 2017: Lagrangian kinematic criterion for the breaking of shoaling waves. J. Phys. Oceanogr., 47, 827833, https://doi.org/10.1175/JPO-D-16-0289.1.

Komen, G. J., K. Hasselmann, and K. Hasselmann, 1984: On the existence of a fully developed wind-sea spectrum. J. Phys. Oceanogr., 14, 1271-1285, https://doi.org/10.1175/ 1520-0485(1984)014<1271:OTEOAF $>2.0 . \mathrm{CO} ; 2$.

Lenain, L., and W. K. Melville, 2017: Measurements of the directional spectrum across the equilibrium-saturation ranges of wind-generated surface waves. J. Phys. Oceanogr., 47, 2123 2138, https://doi.org/10.1175/jpo-d-17-0017.1.

Long, C. E., and D. T. Resio, 2007: Wind wave spectral observations in Currituck Sound, North Carolina. J. Geophys. Res., 112, C05001, https://doi.org/10.1029/2006JC003835.

Longuet-Higgins, M. S., 1988: Mechanisms of wave breaking in deep water. Sea Surface Sound, B. R. Kerman, Ed., Springer, 1-30.

Melville, W. K., 1996: The role of surface-wave breaking in air-sea interaction. Annu. Rev. Fluid Mech., 28, 279-321, https:// doi.org/10.1146/annurev.fl.28.010196.001431.

Perlin, M., W. Choi, and Z. Tian, 2013: Breaking waves in deep and intermediate waters. Annu. Rev. Fluid Mech., 45, 115-145, https://doi.org/10.1146/annurev-fluid-011212-140721.

Phillips, O. M., 1958: The equilibrium range in the spectrum of wind-generated waves. J. Fluid Mech., 4, 426-434, https:// doi.org/10.1017/S0022112058000550.

_ 1985: Spectral and statistical properties of the equilibrium range in wind-generated gravity waves. J. Fluid Mech., 156, 505-531, https://doi.org/10.1017/S0022112085002221.

Pierson, W. J., and L. Moskowitz, 1964: A proposed spectral form for fully developed wind seas based on the similarity theory of S. A. Kitaigorodskii. J. Geophys. Res., 69, 5181-5190, https:// doi.org/10.1029/JZ069i024p05181.

Rapp, R. J., and W. K. Melville, 1990: Laboratory measurements of deep-water breaking waves. Philos. Trans. Roy. Soc. London, 331A, 735-800, https://doi.org/10.1098/rsta.1990.0098.
Resio, D. T., J. H. Pihl, B. A. Tracy, and L. C. Vincent, 2001: Nonlinear energy fluxes and the finite depth equilibrium range in wave spectra. J. Geophys. Res., 106, 6985-7000, https:// doi.org/10.1029/2000JC900153.

C. E. Long, and L. C. Vincent, 2004: Equilibrium-range constant in wind-generated wave spectra. J. Geophys. Res., 109, C01018, https://doi.org/10.1029/2003JC001788.

_ _ _ , and W. Perrie, 2011: The role of nonlinear momentum fluxes on the evolution of directional wind-wave spectra. J. Phys. Oceanogr., 41, 781-801, https://doi.org/ 10.1175/2010JPO4545.1.

Rodríguez, G., and C. G. Soares, 1999: Uncertainty in the estimation of the slope of the high frequency tail of wave spectra. Appl. Ocean Res., 21, 207-213, https://doi.org/ 10.1016/S0141-1187(99)00014-0.

Romero, L., and W. K. Melville, 2010: Airborne observations of fetch-limited waves in the Gulf of Tehuantepec. J. Phys. Oceanogr., 40, 441-465, https://doi.org/10.1175/2009JPO4127.1.

- — - and J. M. Kleiss, 2012: Spectral energy dissipation due to surface wave breaking. J. Phys. Oceanogr., 42, 1421-1444, https://doi.org/10.1175/JPO-D-11-072.1.

Saket, A., W. L. Peirson, M. L. Banner, X. Barthelemy, and M. J. Allis, 2017: On the threshold for wave breaking of twodimensional deep water wave groups in the absence and presence of wind. J. Fluid Mech., 811, 642-658, https://doi.org/ 10.1017/jfm.2016.776.

Schwendeman, M., J. Thomson, and J. R. Gemmrich, 2014: Wave breaking dissipation in a young wind sea. J. Phys. Oceanogr., 44, 104-127, https://doi.org/10.1175/JPO-D-12-0237.1.

Shemer, L., and D. Liberzon, 2014: Lagrangian kinematics of steep waves up to the inception of a spilling breaker. Phys. Fluids, 26, 016601, https://doi.org/10.1063/1.4860235.

Smith, J. M., 2003: Equilibrium ranges in surf zone wave spectra. J. Geophys. Res., 108, 3366, https://doi.org/ 10.1029/2003JC001930.

Song, J.-B., and M. L. Banner, 2002: On determining the onset and strength of breaking for deep water waves. Part I: Unforced irrotational wave groups. J. Phys. Oceanogr., 32, 2541-2558, https://doi.org/10.1175/1520-0485-32.9.2541.

Stokes, G. G., 1880: On the theory of oscillatory waves. Mathematical and Physical Papers, Vol. 1, Cambridge University Press, 197-229.

Toba, Y., 1972: Local balance in the air-sea boundary processes: I. On the growth process of wind waves. J. Oceanogr. Soc. Japan, 28, 109-120, https://doi.org/10.1007/BF02109772.

- 1973: Local balance in the air-sea boundary processes: III. On the spectrum of wind waves. J. Oceanogr. Soc. Japan, 29, 209220, https://doi.org/10.1007/BF02108528.

Toffoli, A., A. V. Babanin, M. Onorato, and T. Waseda, 2010: Maximum steepness of oceanic waves: Field and laboratory. Geophys. Res. Lett., 37, L05603, https://doi.org/10.1029/ 2009GL041771.

Tsagareli, K. N., A. V. Babanin, D. J. Walker, and I. R. Young, 2010: Numerical investigation of spectral evolution of wind waves. Part I: Wind-input source function. J. Phys. Oceanogr., 40, 656-666, https://doi.org/10.1175/2009JPO4345.1.

Tulin, M. P., and M. Landrini, 2001: Breaking waves in the ocean and around ships. Twenty-Third Symposium on Naval Hydrodynamics, National Academies Press, 713745.

Umesh, P. A., P. K. Bhaskaran, K. G. Sandhya, and T. M. B. Nair, 2018: High frequency tail characteristics in the coastal waters off Gopalpur, northwest Bay of Bengal: A nearshore 
modelling study. Pure Appl. Geophys., 175, 2351-2379, https://doi.org/10.1007/s00024-017-1761-1.

Waseda, T., and Coauthors, 2014: Deep water observations of extreme waves with moored and free GPS buoys. Ocean Dyn., 64, 1269-1280, https://doi.org/10.1007/s10236-0140751-4.

Young, I. R., and A. V. Babanin, 2006: Spectral distribution of energy dissipation of wind-generated waves due to dominant wave breaking. J. Phys. Oceanogr., 36, 376-394, https:// doi.org/10.1175/JPO2859.1.

Zakharov, V. E., and N. N. Filonenko, 1967: Energy spectrum for stochastic oscillations of the surface of a liquid. Sov. Phys. Dokl., 11, 811.

Zhao, D., and Y. Toba, 2001: Dependence of whitecap coverage on wind and wind-wave properties. J. Oceanogr., 57, 603-616, https:// doi.org/10.1023/A:1021215904955. 